\title{
Factors influencing the acquisition of tactual random figure discriminations by rhesus monkeys*
}

\author{
KRISTIN R. CARLSON† and ROBERT EIBERGEN \\ Department of Pharmacology, University of Pittsburgh School of Medicine \\ Pittsburgh, Pennsylvania 15261
}

\begin{abstract}
Rhesus monkeys learned to perform two-object random figure discrimination problems by touch. This performance was compared to the visual acquisition of the same problems (reported earlier). In both modalities, choice latencies were shorter to the correct than to the incorrect figure. As compared to visual, however, tactual learning required significantly more trials to criterion, was independent of stimulus figure complexity, and was characterized by significantly shorter choice latencies. Rotation of the figures $180 \mathrm{deg}$ after criterion performance was achieved, yielded partial recognition of the figures visually, but no recognition tactually. These results are explained by different observational or cue-utilization strategies. The majority of the figure is inspected visually, but only the same small section of the perimeter is used tactually. Thus, restricted use of the available cues characterizes tactual performance and is responsible for generating differences in various measures of learning between vision and touch in the monkey.
\end{abstract}

Most investigations of tactual problem solving by monkeys have been primarily designed to measure the effects of neurological damage (e.g., Manzoni \& Ettlinger, 1971; Stamm \& Rosen, 1971; Passingham \& Ettlinger, 1972). Surprisingly little attention has been directed toward the strategies employed by normal monkeys in performing tactual discriminations or toward comparing tactual and visual discrimination ability. Wilson's (1965) work is a notable exception. She examined the acquisition of tactual and visual learning sets as a function of six basic hypotheses used by the monkeys, and later showed the usefulness of this approach in analyzing deficits produced by brain lesions (Oscar \& Wilson, 1966). Although it may be inferred from her work that more trials are required to learn tactual as opposed to visual problems, there has been no systematic investigation of similarities and differences between the two modalities in various measures of problem-solving skill, the question to which the present experiment is directed.

This analysis has been greatly facilitated by the use of random figure objects (Attneave \& Arnoult, 1956). These figures have been used extensively in human discrimination studies (for review see Zusne, 1970), and possess several attributes which make them ideal for discriminations with nonhuman primates as well. For example, an infinite number can be constructed, their metrics can be precisely specified, and they are equally

*This research was supported by Grant MH 20121 from the National Institute of Mental Health to the first author. The excellent technical assistance of $\mathrm{K}$. Bodner, $\mathrm{M}$. Cochenour, $\mathrm{K}$. Erleba, and M. Svegel in the pretraining phase of the experiment is gratefully acknowledged. Portions of this study were presented at the 1974 meeting of the Eastern Psychological Association.

tRequests for reprints should be addressed to Dr. K. R. Carlson, 620 Scaife Hall, University of Pittsburgh, Pittsburgh, Pennsylvania 15261 . unfamiliar to the Ss. Representative figures are shown in Fig. 1.

We recently investigated the visual discrimination performance of rhesus monkeys on a series of two-object random figure problems (Carlson, 1973). First, we
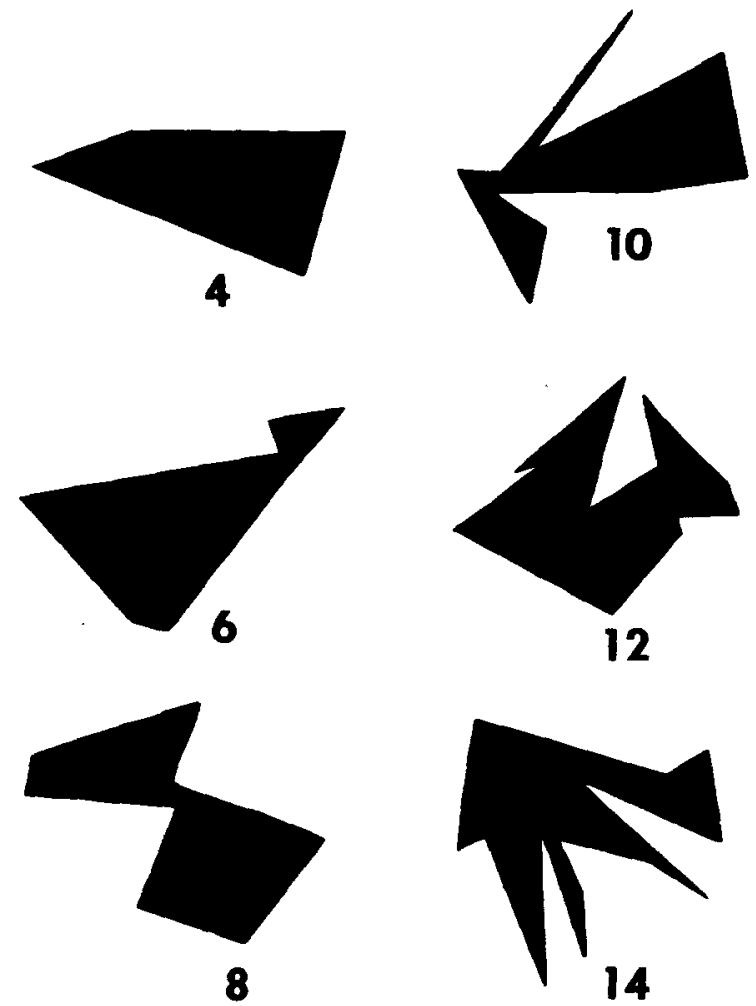

Fig. 1. Examples of the random figures used in this study. The number of sides is indicated beneath each figure. Each problem consisted of two figures with the same number of sides. 


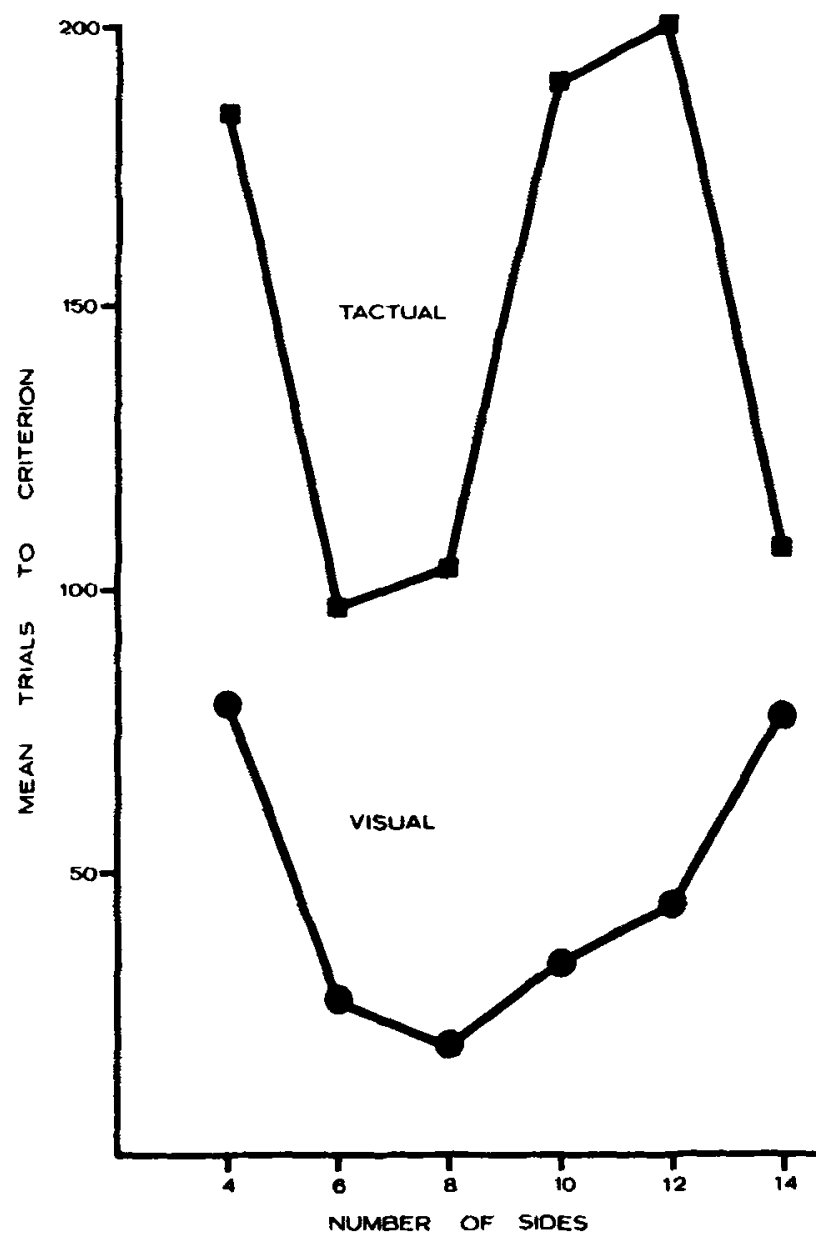

Fig. 2. Mean trials to criterion during training as a function of number of sides in the stimulus figures, for problems learned visually (circles) and tactually (squares).

showed that trials to criterion was a U-shaped function of the complexity, i.e., number of sides, of the figures, in that acquisition was most rapid at intermediate complexity levels. A similar U-shaped function is generated by human observers in various visual tasks using random figures (Crook, 1957; Brown, Hitchcock, \& Michels, 1962; Owen \& Brown, 1970b), suggesting substantial cross-species comparability. Since errors made by human Ss in a tactual random figure task also follow a U-shaped function (Owen \& Brown, 1970b), it is reasonable to speculate that this is a phenomenon not restricted to a particular sense modality which might be observed in the monkey as well.

Secondly, we found that average choice latencies were longer to the incorrect than to the correct object, confirming other visual studies in the rat (Feldman, 1953; Mahut, 1954) and monkey (Milner \& Ettlinger, 1972). In the present experiment, we also measured latencies to determine whether this relationship obtained in tactual performance as well. Comparing the two modalities, we expected tactual to be much longer than visual latencies, based on data from humans (Owen \& Brown, 1970a,b).
Finally, we found that when the figures were rotated $180 \mathrm{deg}$ after a problem had been learned to criterion, visual performance was only partially disrupted and there was some recognition of the problem. Since changes in orientation adversely affect tactual more than visual recognition in humans (Goodnow, 1969), we were interested in testing this effect in the monkey.

In short, our objective was to measure several dependent variables during the tactual acquisition of the same random figure problems which had been learned visually in order to evaluate similarities and differences in the characteristics of the learning process in the two sensory modalities.

\section{METHOD}

\section{Subjects}

Four mature male rhesus monkeys (Macaca mulatta) served as Ss. One had participated in a previous experiment (Carlson, 1973), in which he learned these discrimination problems visually. The other three had no experience with random figures. They were housed individually, had ad lib access to water, and were fed Purina monkey chow twice daily.

\section{Apparatus}

For each experimental session, $\mathrm{S}$ was seated in a temporary restraint chair (Carlson, 1972) which was fitted with opaque neck plates and turret to prevent him from seeing the discriminanda. He could deliver the food reward to his mouth through a small hole in the neck plate underneath his chin. A 23-cm wide WGTA-type stimulus tray was attached to the front of the chair and was accessible to $S$ 's hands through a $5.5-\mathrm{cm}$ high $\times 21-\mathrm{cm}$ wide slot which could be closed by a sliding door. The two foodwells were $11 \mathrm{~cm}$ apart and $5 \mathrm{~cm}$ from the edge of the tray closest to $S$.

The discriminanda were the same random figures used in the visual discrimination experiment (Carlson, 1973), $30 \mathrm{~cm}^{2}$ in area, $1.2 \mathrm{~cm}$ thick, with $4,6,8,10,12$, or 14 sides on the perimeter (Fig. 1). Each figure was mounted flat on a $10 \times 10 \mathrm{~cm}$ piece of thin Plexiglas which slid under flanges positioned at both sides of each foodwell. A locking device prevented $\mathrm{S}$ from pulling either figure toward him and from pushing both away from him simultaneously, thereby ensuring that he could uncover only one foodwell at a time.

Latencies were recorded manually on a Lafayette 1/100 $\mathrm{sec}$ stop clock. Raisins were used as rewards.

\section{Procedure}

Ss underwent extensive pretraining during which their view of the stimulus tray was progressively reduced and the size and location of the hole in the neckplate gradually changed until they were reliably performing two-object discriminations by touch alone. Ss were permitted to use either or both hands throughout the experiment.

Training consisted of a series of successive two-object random figure discriminations, two problems at each of the six sidedness levels. Both objects in each problem had the same number of sides and were presented in the same orientation on each training trial. This set of problems corresponds to the first half of the series presented visually, and the training procedure was essentially the same. The position of the correct object was varied pseudorandomly with an equal number of right and left positions being correct during each daily session. Latencies were measured from the time the sliding access door was opened to the moment $S$ pushed one figure far enough to uncover a foodwell. The intertrial interval was $20 \mathrm{sec}$. Ss were given 40 noncorrection trials per day, to a criterion of $36 / 40$ correct choices. After criterion performance was attained on each 
problem, both stimulus figures were rotated $180 \mathrm{deg}$ and an additional 20 trials were given. If $S$ did not reach criterion within 480 trials, that problem was discontinued; this was necessary on only three occasions.

\section{RESULTS}

Since the tactual problem series comprised the first half of the visual series reported in Carlson (1973), that first half was reanalyzed in order to provide a more valid performance comparison. The reanalyzed data presented here did not differ significantly in any aspect from the data generated by the entire series reported earlier.

Although the tactual problems could be learned, it is clear from Fig. 2 that Ss required many more trials to criterion tactually than visually (Mann-Whitney U-test, $\mathrm{z}=-3.58, \mathrm{p}<.001)$. In addition, there was greater variance tactually than visually as reflected in the standard error of the mean computed across all sidedness levels (tactual $= \pm 24.3$, visual $= \pm 10.5$ ). Furthermore, the significant U-shaped function between sidedness level and trials found in the visual modality was not replicated tactually (Friedman analysis of variance, $\chi^{2}=4.5$, ns). Apparently, the complexity of the total figure, as estimated by the number of sides, was not a determinant of S's performance tactually.

The final point concerning trials to criterion is that visual experience with these problems did not seem to assist in their subsequent acquisition tactually. The $S$ which had learned these problems visually required an average of 188.7 trials to criterion, whereas the average of the other Ss was 133.2 trials. Due to the high variability, the apparent difference in the direction of inferior tactual performance by the visually experienced $S$ was not significant (Mann-Whitney U-test, $U=34$, ns).

With respect to the latency measures, Fig. 3 shows that tactual latencies were consistently shorter than visual latencies, both when the correct figure was chosen (Mann-Whitney U-test, $z=-5.25, \mathrm{p}<.001$ ) and when the incorrect figure was chosen (Mann-Whitney U-test, $\mathrm{z}=5.56, \mathrm{p}<.001)$. The relationship shown so strikingly in the visual data, i.e., incorrect latencies longer than correct latencies, was replicated in the tactual data (Wilcox on test, $T=68, p<.02$ ). Thus, when using the tactual modality, Ss show shorter latencies when choosing the correct as opposed to the incorrect figure, and in general choose much more rapidly than when solving the same problems visually.

The data from the rotation test shown in Fig. 4 are particularly interesting. When performing these discriminations visually, there was partial recognition of the stimulus figures when they were rotated $180 \mathrm{deg}$, since errors on the 20 rotation trials were significantly fewer than on the first 20 trials of that problem (Wilcoxon test, $\mathrm{T}=0, \mathrm{p}<.01$ ). Tactually, however, there was no transfer to the rotated figures; Ss made as many errors on the rotation trials as they had on the first 20 trials (Wilcoxon test, $\mathrm{T}=125.5$, ns), in essence treating the rotated figures as an entirely new problem.

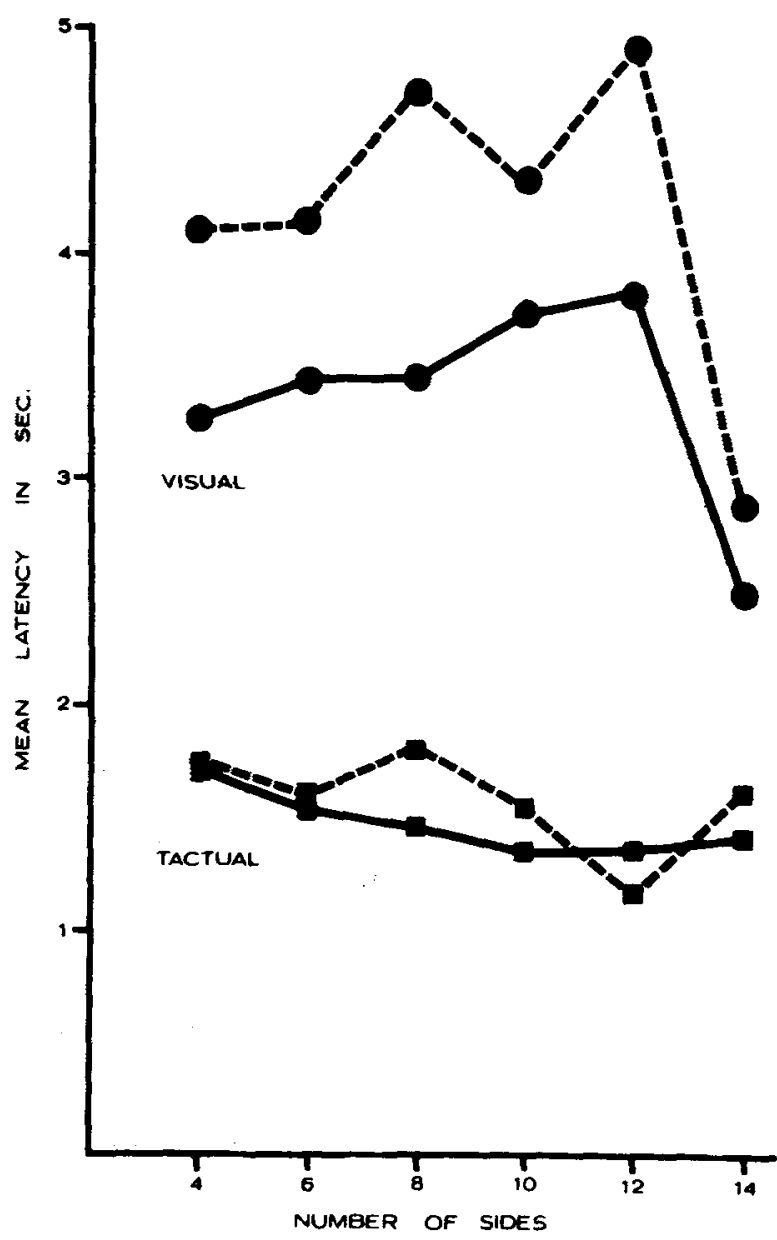

Fig. 3. Mean latency to choice during training as a function of number of sides in the stimulus figures, when the correct figure was chosen (solid lines) and when the incorrect figure was chosen (dashed lines), in visual (circles) and tactual (squares) modalities.

\section{DISCUSSION}

The fact that more trials were required to acquire these discriminations tactually than visually is intuitively sensible and consistent with the relative difficulty of establishing tactual as opposed to visual learning sets (Wilson, 1965). It was also predictable that prior visual experience would not assist in tactual learning of the same problems, on the basis of a general failure to observe cross-modal transfer in similar situations (for review see Frampton, Milner, \& Ettlinger, 1973).

Virtually the only similarity between the visual and tactual data was the fact that choice latencies were significantly longer to the incorrect than to the correct figure in both modalities. This phenomenon has been reported before for vision (Carlson, 1973; Milner \& Ettlinger, 1972), and the present experiment suggests that hesitation before choosing the incorrect object is a modality-nonspecific characteristic of primate discrimination learning. 


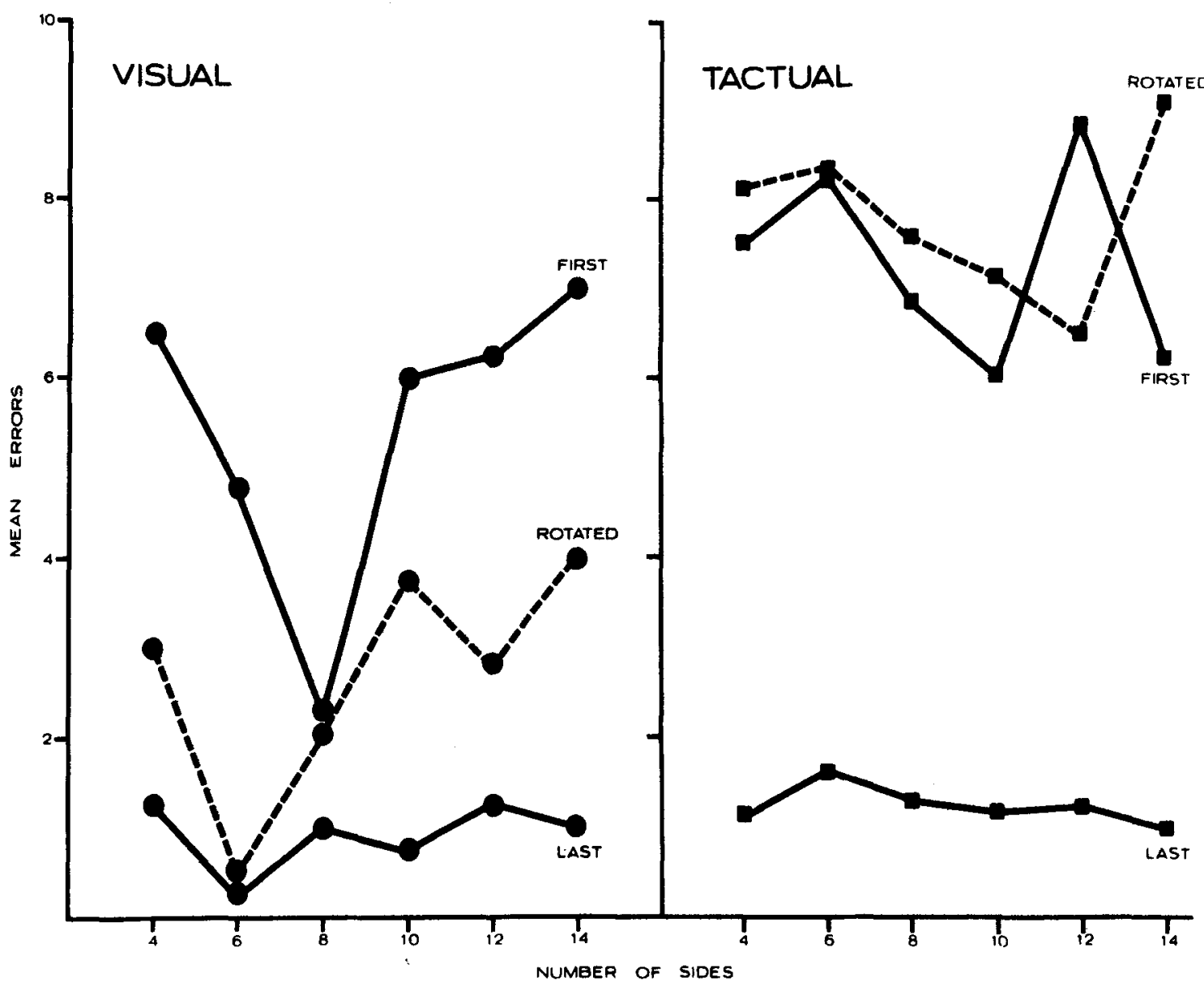

Fig. 4. Mean errors as a function of number of sides in the stimulus figures, on the first 20 trials of a problem (First), on the last 20 trials (Last), and on the subsequent 20 trials in which both objects were rotated 180 deg (Rotated). Left panel shows visual and right panel shows tactual modalities.

The most striking impression from the data, however, is one of large differences between the two modalities, such as the absence of a U-shaped function between tactual trials to criterion and number of sides, greater variability in trials to criterion tactually, shorter tactual latencies, and the lack of tactual recognition when the figures were rotated. We suggest that all these effects are explicable by Ss' observational strategies, i.e., the manner in which they touched the objects while learning tactual discriminations.

Whether using one or both hands, $S$ would touch only a limited section of the perimeter of a figure. There was inter-S variability in the typical location of this section, i.e., some Ss attended to the edge nearest to them and others to the far edge, but the important point is that Ss were performing the discrimination on the basis of fewer cues than were contained in the entire figures. They gave no impression of palpating the figures in order to form mental images of their shapes, but rather of finding some differentiating cue and using that single aspect in discriminating between the figures.

The use of this strategy would explain the lack of any clear-cut relationship between sideness level and difficulty of the discrimination. Since Ss were not observing the entire figure, the number of sides was not a consistent factor in their performance; more crucial was the presence of a convenient cue for differentiating the tigures. Une would also expect greater variability in the number of trials necessary to find and use successfully a single salient cue, as opposed to visual use of the figure's entire configuration. This was, in fact, the case.

The necessity of using only a small part of the figures undoubtedly contributed to the surprisingly short, 1 to $2-\mathrm{sec}$, tactual latencies as well. Human observers require an average of 14 to $38 \mathrm{sec}$, depending on the nature of the problem (Owen \& Brown, 1970a,b). The most logical explanation for this discrepancy is that the human Ss more thoroughly explored the figures than did our monkeys.

These same differences in observational strategies, as well as S's restrained position in constant physical proximity to the stimulus figures, could well be responsible for shorter tactual than visual latencies in the present experiment. The sequence of actions required of $\mathrm{S}$ in the tactual task was to reach out approximately 
$10 \mathrm{~cm}$, search for a prominant part of one figure, and push a figure away from him, all of which could be accomplished very rapidly. The situation when performing these discriminations visually in a WGTA was quite different: $S$ was often in a remote area of the cage when the opaque screen was lifted and the latency measure begun, and he had to move forward, observe the figures, and reach out to displace one of them. We therefore think that both aspects of the tactual task, restraint in physical proximity and utilization of fewer cues, contributed to the shorter tactual latencies. The relative influence of each factor can be determined only by additional experimentation, for example, by administering these problems visually while $S$ is restrained in the same manner as in the present tactual paradigm.

The importance of restricted cue utilization in the tactual modality is undeniable in explaining the error data from the rotation test. Tactual Ss reacted as if they had had no experience with the figures after they were rotated $180 \mathrm{deg}$, which is understandable when one considers that the section of the figures they were consistently using was now on the other side of each figure. Thus, the figures felt completely different, and $\mathrm{Ss}$ treated them as a new problem to be solved. Visual Ss, on the other hand, although not showing perfect transfer to the rotated figures, at the very least indicated by their error scores that they were attending to a larger proportion of the shape of the stimulus figures than were Ss using the tactual modality. These findings not only extend Goodnow's (1969) observation that changes in orientation disrupt tactual more than visual recognition for human Ss, but provide an explanation, for the monkey, in terms of differing observational strategies.

In summary, the observed differences between the visual and tactual modalities were generated by two factors. One, whose importance is yet to be fully assessed, was the physical configuration of the testing space, which probably affected the latency measure. The other was the observational strategy adopted by the tactual Ss, which limited their perception to a few salient cues in the stimulus figures. This strategy could be changed by rotating the figures throughout training, thereby forcing $S$ to palpate them more completely. This technique greatly increases the difficulty of visual discriminations for monkeys (Hicks, 1967), and we suspect it would produce insoluable tactual problems. Nonetheless, the present comparison of visual and tactual discrimination performance represents initial evidence that different cue-utilization strategies are responsible for generating large differences in various measures of learning in the two modalities.

\section{REFERENCES}

Attneave, $F$.q \& Amoult, M. The quantitative study of shape and pattern perception. Psychological Bulletin, 1956, 53, 452-471.

Brown, D., Hitchcock, L., \& Michels, $K$. Quantitative studies in form perception: An evaluation of the role of selected stimulus parameters in the visual discrimination performance of human subjects. Perceptual \& Motor Skills, 1962, 14, 519-529.

Carlson, K. A temporary restraint chair for monkeys. Physiology \& Behavior, $1972,9,493-494$.

Carlson, K. Visual discrimination learning of random figure problems by rhesus monkeys. Animal Learning \& Behavior, $1973,1,33-37$.

Crook, $M$. Facsimile-generated analogues for instrumental displays. In J. Wulfeck \& J. Taylor (Eds.), Form discrimination as related to military problems. Washington, D.C.: NAS-NRC, 1957, Pp. 85-98.

Feldman, $R$. The specificity of the fixated response in the rat. Journal of Comparative \& Physiological Psychology, 195 3, 46, 487-492.

Frampton, G., Milner, A., \& Ettlinger, G. Cross-modal transfer between vision and touch of go, no-go discrimination learning in the monkey. Neuropsychologia, 1973, 11, 231-233.

Goodnow. J. Eye and hand: Differential sampling of form and orientation properties. Neuropsychologia, 1969, 7, 365-373.

Hicks, L. Effects of stimulus rotation on discrimination learning by monkeys. Psychonomic Science, 1967, 9, 57-58.

Mahut, H. The effect of stimulus position on visual discrimination by the rat. Canadian Journal of Psychology, 1954, 8, 130-138,

Manzoni, T., Ettlinger, G. Impairment of tactile discrimination performance in monkeys with tectal damage. Neuropsychologia, 1971, 9, 289-292.

Milner, A., \& Ettlinger, G. Response latencies in go, no-go discrimination performance by monkeys. Neuropsychologia, $1972,10,375-378$.

Oscar, M., \& Wilson, M. Tactual and visual discrimination learning in monkeys with frontal lesions. Journal of Comparative \& Physiological Psychology, 1966, 62, 108-114.

Owen, D., \& Brown, D. Visual and tactual form complexity: A psychophysical approach to perceptual equivalence. Perception \& Psychophysics, 1970a, 7, 225-228.

Owen, D., \& Brown, D. Visual and tactual form discrimination: Psychophysical comparison within and between modalities. Perception \& Psychophysics, 1970b, 7, 302-306.

Passingham, R., \& Ettlinger, $\dot{G}$. Tactile discrim ination learning after selective prefrontal ablations in monkeys (Macaca mulatta). Neuropsychologia, 1972, 10 . 17-26.

Stamm, J., \& Rosen, S. Learning on somesthetic discrimination and reversal tasks by monkeys with epileptogenic implants in anteromedial temporal cortex. Neuropsychologia, 1971, 9 , 185-194.

Wilson, M. Tactual discrimination learning in monkeys. Neuropsychologia, 1965, 3, 353-361.

Zusne, L. Visual perception of form. New York : Academic Press, 1970 .

(Received for publication December 2, 1973; revision received February $5,1974$. ) 